\title{
ON HARMONIC WEAK MAASS FORMS OF HALF INTEGRAL WEIGHT
}

\author{
BUMKYU CHO AND YOUNGJU CHOIE
}

(Communicated by Kathrin Bringmann)

\begin{abstract}
Since Zwegers found a connection between mock theta functions and harmonic weak Maass forms, this subject has been of vast research interest. In this paper, we obtain isomorphisms among the space $H_{k+\frac{1}{2}}^{+}\left(\Gamma_{0}(4 m)\right)$ of (scalar valued) harmonic weak Maass forms of half integral weight whose Fourier coefficients are supported on suitable progressions, the space $H_{k+\frac{1}{2 x 1}, \bar{\rho}_{L}}$ of vector valued ones, and the space $\mathrm{x} 1 \widehat{J}_{k+1, m}^{\text {cusp }}$ of certain harmonic MaassJacobi forms of integral weight:

$$
H_{k+\frac{1}{2}}^{+}\left(\Gamma_{0}(4 m)\right) \simeq H_{k+\frac{1}{2}, \bar{\rho}_{L}} \simeq \widehat{\mathbb{J}}_{k+1, m}^{c u s p}
$$

for $k$ odd and $m=1$ or a prime. This is an extension of a result developed by Eichler and Zagier, which shows that

$$
M_{k+\frac{1}{2}}^{+}\left(\Gamma_{0}(4 m)\right) \simeq M_{k+\frac{1}{2}, \bar{\rho}_{L}} \simeq J_{k+1, m} .
$$

Here $M_{k+\frac{1}{2}}^{+}\left(\Gamma_{0}(4 m)\right), M_{k+\frac{1}{2}, \bar{\rho}_{L}}$ and $J_{k+1, m}$ are the Kohnen plus space of (scalar valued) modular forms of half integral weight, the space of vector valued ones, and the space of Jacobi forms of integral weight, respectively. To extend the result, another approach is necessary because the argument by Eichler and Zagier depends on the dimension formulas for the spaces of holomorphic modular forms, but the dimensions for the spaces of harmonic weak Maass forms are not finite. Our proof relies on some nontrivial properties of the Weil representation.
\end{abstract}

\section{Introduction AND StATEMEnt of A RESUlt}

Let $k$ be an integer and $m$ a positive integer. Denote by $M_{k+\frac{1}{2}}\left(\Gamma_{0}(4 m)\right)$ the space of holomorphic modular forms of weight $k+\frac{1}{2}$ for $\Gamma_{0}(4 m)$. Further define a subspace $M_{k+\frac{1}{2}}^{+}\left(\Gamma_{0}(4 m)\right)$ of $M_{k+\frac{1}{2}}\left(\Gamma_{0}(4 m)\right)$ by

$$
M_{k+\frac{1}{2}}^{+}\left(\Gamma_{0}(4 m)\right):=\left\{f \in M_{k+\frac{1}{2}}\left(\Gamma_{0}(4 m)\right) \mid c_{f}(n)=0 \text { unless }(-1)^{k} n \equiv \square \bmod 4 m\right\} .
$$

Let $L$ be the lattice $2 m \mathbb{Z}$ equipped with the quadratic form $Q(x)=x^{2} / 4 m$. Then its dual $L^{\prime}$ equals $\mathbb{Z}$. Let $\rho_{L}$ denote the Weil representation associated to the discriminant form $\left(L^{\prime} / L, Q\right)$, and let $\bar{\rho}_{L}$ be its dual representation. Denote by

Received by the editors January 31, 2011 and, in revised form, June 6, 2011 and November 10, 2011.

2010 Mathematics Subject Classification. Primary 11F11, 11F30; Secondary 11F37, 11F50.

The first author was partially supported by the Dongguk University Research Fund of 2012 and NRF 2010-0008426.

The second author was partially supported by NRF-2011-0008928, NRF-2011-0030749 and NRF- 2008-0061325. 
$M_{k+\frac{1}{2}, \rho_{L}}$ the space of $\mathbb{C}\left[L^{\prime} / L\right]$-valued holomorphic modular forms of weight $k+\frac{1}{2}$ and type $\rho_{L}$. Then Eichler and Zagier [7, Theorems 5.1, 5.4, and 5.6] obtained the isomorphisms

$$
M_{k+\frac{1}{2}}^{+}\left(\Gamma_{0}(4 m)\right) \simeq M_{k+\frac{1}{2}, \bar{\rho}_{L}} \simeq J_{k+1, m}
$$

for $k$ odd and $m=1$ or a prime; here $J_{k+1, m}$ is the space of Jacobi forms of weight $k+1$ and index $m$ on the full modular group $\Gamma(1)$.

Our result extends these isomorphisms to the spaces of harmonic weak Maass forms (Theorem 1 and Theorem 2): if $k$ is odd and $m=1$ or a prime,

$$
H_{k+\frac{1}{2}}^{+}\left(\Gamma_{0}(4 m)\right) \simeq H_{k+\frac{1}{2}, \bar{\rho}_{L}} \simeq \widehat{\mathbb{J}}_{k+1, m}^{c u s p},
$$

where $H_{k+\frac{1}{2}}^{+}\left(\Gamma_{0}(4 m)\right), H_{k+\frac{1}{2}, \bar{\rho}_{L}}$, and $\widehat{\mathbb{J}}_{k+1, m}^{c u s p}$ are the spaces consisting of the corresponding harmonic ones (see Section 2). To prove this result, another approach is necessary because Eichler and Zagier's argument depends on the dimension formulas for the spaces of holomorphic modular forms, but the dimensions for the spaces of harmonic weak Maass forms are infinite (see the proof of [7, Theorem 5.6]). Our proof relies on some nontrivial properties of the Weil representation (see Section 3).

To state our main results more precisely, let $H_{k+\frac{1}{2}}\left(\Gamma_{0}(4 m)\right)$ be the space of harmonic weak Maass forms of weight $k+\frac{1}{2}$ for $\Gamma_{0}(4 m)$ where $k \in \mathbb{Z}$ and $m=1$ or a prime (see Section 2.1). Then it is known (see, for instance, 5]) that $f \in$ $H_{k+\frac{1}{2}}\left(\Gamma_{0}(4 m)\right)$ has a unique decomposition $f=f^{+}+f^{-}$, where

$$
\begin{aligned}
f^{+}(\tau) & =\sum_{n \gg-\infty} c_{f}^{+}(n) q^{n}, \\
f^{-}(\tau) & =\sum_{n<0} c_{f}^{-}(n) \Gamma\left(\frac{1}{2}-k, 4 \pi|n| y\right) q^{n}
\end{aligned}
$$

with the incomplete gamma function $\Gamma(a, y)=\int_{y}^{\infty} e^{-t} t^{a-1} d t$. Now we define the subspace

$$
H_{k+\frac{1}{2}}^{+}\left(\Gamma_{0}(4 m)\right):=\left\{f \in H_{k+\frac{1}{2}}\left(\Gamma_{0}(4 m)\right) \mid c_{f}^{ \pm}(n)=0 \text { unless }(-1)^{k} n \equiv \square \bmod 4 m\right\} .
$$

Let $H_{k+\frac{1}{2}, \rho_{L}}$ be the space of $\mathbb{C}\left[L^{\prime} / L\right]$-valued harmonic weak Maass forms of weight $k+\frac{1}{2}$ and type $\rho_{L}$ (see Section 2.2). Denote the standard basis elements of the group algebra $\mathbb{C}\left[L^{\prime} / L\right]$ by $\mathfrak{e}_{\gamma}$ for $\gamma \in L^{\prime} / L$. Suppose that the discriminant form $\left(L^{\prime} / L, Q\right)$ is given by $(\mathbb{Z} / 2 m \mathbb{Z}, Q)$, where $Q(\gamma)=\gamma^{2} / 4 m$ for $\gamma \in \mathbb{Z} / 2 m \mathbb{Z}$ with the signature $\left(b^{+}, b^{-}\right)$; then the level of $L$ equals $4 m$, and $b^{+}-b^{-} \equiv 1 \bmod 8$. For example, if we take

$$
L=\left\{X=\left(\begin{array}{cc}
b & -a / m \\
c & -b
\end{array}\right) \in \operatorname{Mat}_{2}(\mathbb{Q}) \mid a, b, c \in \mathbb{Z}\right\}
$$

with $Q(X)=-m \operatorname{det}(X)$, then $\left(b^{+}, b^{-}\right)=(2,1)$ and

$$
L^{\prime}=\left\{X=\left(\begin{array}{cc}
b / 2 m & -a / m \\
c & -b / 2 m
\end{array}\right) \in \operatorname{Mat}_{2}(\mathbb{Q}) \mid a, b, c \in \mathbb{Z}\right\} .
$$

For a given $f \in H_{k+\frac{1}{2}}^{+}\left(\Gamma_{0}(4 m)\right)$, we define a $\mathbb{C}\left[L^{\prime} / L\right]$-valued function $F=$ $\sum_{\gamma \in \mathbb{Z} / 2 m \mathbb{Z}} F_{\gamma} \mathfrak{e}_{\gamma}$ by

$$
F_{\gamma}(\tau):=\frac{1}{s(\gamma)} \sum_{\substack{n \in \mathbb{Z} \\(-1)^{k} \equiv \gamma^{2} \bmod 4 m}} c_{f}(n, y / 4 m) q^{n / 4 m}
$$


where $c_{f}(n, y):=c_{f}^{+}(n)+c_{f}^{-}(n) \Gamma\left(\frac{1}{2}-k, 4 \pi|n| y\right)$, and $s(\gamma)=1$ if $\gamma \equiv 0, m \bmod 2 m$, and 2 otherwise.

Theorem 1. With the notation as above we have the following:

(1) If $k$ is even, then the map $f \mapsto F$ defines an isomorphism from $H_{k+\frac{1}{2}}^{+}\left(\Gamma_{0}(4 m)\right)$ onto $H_{k+\frac{1}{2}, \rho_{L}}$.

(2) If $k$ is odd, then the map $f \mapsto F$ defines an isomorphism from $H_{k+\frac{1}{2}}^{+}\left(\Gamma_{0}(4 m)\right)$ onto $H_{k+\frac{1}{2}, \bar{\rho}_{L}}$.

Remark 1. (1) For a given vector valued modular form $F=\sum_{\gamma} F_{\gamma} \mathfrak{e}_{\gamma}$ the map $F \mapsto f$ will be the inverse isomorphism, where $f(\tau):=\sum_{\gamma} F_{\gamma}(4 m \tau)$.

(2) Applying the same argument in the proof of Theorem 10 we can see that

$$
M_{k+\frac{1}{2}}^{!+}\left(\Gamma_{0}(4 m)\right) \simeq M_{k+\frac{1}{2}, \rho_{L}}^{!}
$$

for $k$ even, and similarly for $k$ odd. Here $M_{k+\frac{1}{2}}^{!+}\left(\Gamma_{0}(4 m)\right)$ denotes the Kohnen space of the weakly holomorphic forms of half integral weight, and $M_{k+\frac{1}{2}, \rho_{L}}^{!}$the space of weakly holomorphic vector valued modular forms.

(3) This kind of result for weakly holomorphic modular forms of integral weight is proved by Bruinier and Bundschuh [4]: Let $p$ be an odd prime. We write $M_{k}^{!}\left(\Gamma_{0}(p), \chi_{p}\right)$ for the space of weakly holomorphic modular forms of weight $k$ for $\Gamma_{0}(p)$ with Nebentypus $\chi_{p}(\cdot)=(\dot{\bar{p}})$. For $\epsilon \in\{ \pm 1\}$ we define the subspace

$$
M_{k}^{! \epsilon}\left(\Gamma_{0}(p), \chi_{p}\right):=\left\{f \in M_{k}^{!}\left(\Gamma_{0}(p), \chi_{p}\right) \mid c_{f}(n)=0 \text { if } \chi_{p}(n)=-\epsilon\right\} .
$$

Let $L$ be the lattice so that the discriminant group $L^{\prime} / L$ is isomorphic to $\mathbb{Z} / p \mathbb{Z}$. On $L^{\prime} / L$ the quadratic form is equivalent to $Q(x)=\alpha x^{2} / p$ for some $\alpha \in \mathbb{Z} / p \mathbb{Z}-\{0\}$. Then Bruinier and Bundschuh [4, Theorem 5] showed that $M_{k}^{!} \epsilon\left(\Gamma_{0}(p), \chi_{p}\right)$ is isomorphic to $M_{k, \rho_{L}}^{!}$for $\epsilon=\chi_{p}(\alpha)$. Their argument can be applied to the spaces of harmonic weak Maass forms of integral weight (see [6, Theorem 1.2]).

Next we show that the spaces in Theorem 1-(2) are isomorphic to the space of harmonic Maass-Jacobi forms recently developed by Bringmann and Richter 2 .

Let $L$ be the lattice $2 m \mathbb{Z}$ equipped with the positive definite quadratic form $Q(x)=x^{2} / 4 m$. Then the space $J_{k, m}$ of Jacobi forms of weight $k$ and index $m$ is isomorphic to the space $M_{k-\frac{1}{2}, \bar{\rho}_{L}}$ of $\mathbb{C}\left[L^{\prime} / L\right]$-valued holomorphic modular forms of weight $k-\frac{1}{2}$ and type $\bar{\rho}_{L}$ (see [7. Theorem 5.1]). Recently, Bringmann and Richter [2] introduced harmonic Maass-Jacobi forms, which include the classical Jacobi forms. Let $\hat{\mathbb{J}}_{k, m}^{c u s p}$ be the space of certain harmonic Maass-Jacobi forms

$$
\phi(\tau, z)=\sum_{\substack{n, r \in \mathbb{Z} \\ D \ll \infty}} c^{+}(n, r) q^{n} \zeta^{r}+\sum_{\substack{n, r \in \mathbb{Z} \\ D>0}} c^{-}(n, r) \Gamma\left(\frac{3}{2}-k, \frac{\pi D y}{m}\right) q^{n} \zeta^{r}
$$

of weight $k$ and index $m$ (see Section 2.3), where $D=r^{2}-4 n m, q=e^{2 \pi i \tau}$ and $\zeta=e^{2 \pi i z}$. By the transformation property of harmonic Maass-Jacobi forms [2, Definition 3], one can deduce that if $r^{\prime} \equiv r \bmod 2 m$ and $D^{\prime}=D$ with $D^{\prime}:=$ $r^{\prime 2}-4 n^{\prime} m$, then

$$
c^{ \pm}\left(n^{\prime}, r^{\prime}\right)=c^{ \pm}(n, r), \quad \Gamma\left(\frac{3}{2}-k, \frac{\pi D^{\prime} y}{m}\right)=\Gamma\left(\frac{3}{2}-k, \frac{\pi D y}{m}\right) .
$$


Hence, we can express $\phi(\tau, z)$ as a linear combination of the theta functions of the form

$$
\phi(\tau, z)=\sum_{\mu \in \mathbb{Z} / 2 m \mathbb{Z}} h_{\mu}(\tau) \theta_{m, \mu}(\tau, z)
$$

where

$$
\begin{aligned}
h_{\mu}(\tau):= & \sum_{N \gg-\infty} c^{+}\left(\frac{N+r^{2}}{4 m}, r\right) q^{N / 4 m} \\
& +\sum_{N<0} c^{-}\left(\frac{N+r^{2}}{4 m}, r\right) \Gamma\left(\frac{3}{2}-k,-\frac{\pi N y}{m}\right) q^{N / 4 m}
\end{aligned}
$$

with any $r \in \mathbb{Z}, r \equiv \mu \bmod 2 m$, and

$$
\theta_{m, \mu}(\tau, z):=\sum_{\substack{r \in \mathbb{Z} \\ r \equiv \mu \bmod 2 m}} q^{r^{2} / 4 m} \zeta^{r} .
$$

Using the same argument as in [7, Theorem 5.1], the $2 m$-tuple $\left(h_{\mu}\right)_{\mu(2 m)}$ satisfies the desired transformation formula for vector valued harmonic weak Maass forms. Now it remains to show that $\Delta_{k-\frac{1}{2}} h_{\mu}=0$. By definition, $\phi(\tau, z)$ vanishes under the action of the Casimir element $C^{k, m}$ (see [2, p. 2305]), and the action of $C^{k, m}$ on the functions in $\hat{\mathbb{J}}_{k, m}^{c u s p}$ agrees with that of

$$
C^{k, m}=-2 \Delta_{k-\frac{1}{2}}+\frac{(\tau-\bar{\tau})^{2}}{4 \pi i m} \partial_{\bar{\tau} z z}
$$

(see 2, Proof of Lemma 1]). Using the fact that $\theta_{m, \mu}(\tau, z)$ is in the heat kernel, that is,

$$
\left(\partial_{\tau}-\frac{1}{8 \pi i m} \partial_{z z}\right)\left(\theta_{m, \mu}\right)=0
$$

one can conclude by a direct computation that

$$
C^{k, m}(\phi)=0 \Longrightarrow \Delta_{k-\frac{1}{2}}\left(h_{\mu}\right)=0
$$

for all $\mu \in \mathbb{Z} / 2 m \mathbb{Z}$, which shows that

$$
\hat{\mathbb{J}}_{k, m}^{c u s p} \simeq H_{k-\frac{1}{2}, \bar{\rho}_{L}} .
$$

Hence, we get the following theorem:

Theorem 2. Let $k$ be even, and $m=1$ or a prime. Then

$$
\hat{\mathbb{J}}_{k, m}^{c u s p} \simeq H_{k-\frac{1}{2}}^{+}\left(\Gamma_{0}(4 m)\right) .
$$

Remark 2. (1) In the case of $k$ odd, $M_{k-\frac{1}{2}}^{+}\left(\Gamma_{0}(4 m)\right)$ is isomorphic to the space of skew holomorphic Jacobi forms of weight $k$ and index $m$. So one can guess that there must be a similar isomorphism as above when $k$ is odd. To do that, we need to introduce a new definition of Maass Jacobi forms which include skew holomorphic Jacobi forms. We hope this can be done by following and modifying the work of Bringmann and Richter [2]. 
(2) In the argument of the proof, the growth condition doesn't matter. Namely, if we redefine $H_{k-\frac{1}{2}, \bar{\rho}_{L}}$ and $H_{k-\frac{1}{2}}^{+}\left(\Gamma_{0}(4 m)\right)$ so that they have at most linear exponential growth at cusps, then, for $k$ even and $m=1$ or a prime,

$$
\hat{\mathbb{J}}_{k, m} \simeq H_{k-\frac{1}{2}, \bar{\rho}_{L}} \simeq H_{k-\frac{1}{2}}^{+}\left(\Gamma_{0}(4 m)\right),
$$

where $\hat{\mathbb{J}}_{k, m}$ is the corresponding larger space (see Section 2.3).

\section{Preliminaries}

2.1. Scalar valued modular forms. Let $\mathbb{H}$ be the complex upper half plane, and consider a point $\tau=x+i y \in \mathbb{H}$ with $x, y \in \mathbb{R}$. Let $k \in \frac{1}{2} \mathbb{Z}-\mathbb{Z}$, and $m$ be a positive integer. Put $\varepsilon_{d}:=\left(\frac{-1}{d}\right)^{\frac{1}{2}}$.

Recall that weakly holomorphic modular forms of weight $k$ for $\Gamma_{0}(4 m)$ are holomorphic functions $f: \mathbb{H} \rightarrow \mathbb{C}$ satisfying the following conditions:

(i) For all $\left(\begin{array}{ll}a & b \\ c & d\end{array}\right) \in \Gamma_{0}(4 m)$ we have

$$
f\left(\frac{a \tau+b}{c \tau+d}\right)=\left(\frac{c}{d}\right) \varepsilon_{d}^{-2 k}(c \tau+d)^{k} f(\tau) .
$$

(ii) $f$ has a Fourier expansion of the form

$$
f(\tau)=\sum_{\substack{n \in \mathbb{Z} \\ n \gg-\infty}} c_{f}(n) q^{n},
$$

and analogous conditions are required at all cusps.

A smooth function $f: \mathbb{H} \rightarrow \mathbb{C}$ is called a harmonic weak Maass form of weight $k$ for $\Gamma_{0}(4 m)$ if it satisfies the following conditions:

(i) For all $\left(\begin{array}{ll}a & b \\ c & d\end{array}\right) \in \Gamma_{0}(4 m)$ we have

$$
f\left(\frac{a \tau+b}{c \tau+d}\right)=\left(\frac{c}{d}\right) \varepsilon_{d}^{-2 k}(c \tau+d)^{k} f(\tau) .
$$

(ii) $\Delta_{k} f=0$, where $\Delta_{k}$ is the weight $k$ hyperbolic Laplace operator defined by

$$
\Delta_{k}:=-y^{2}\left(\frac{\partial^{2}}{\partial x^{2}}+\frac{\partial^{2}}{\partial y^{2}}\right)+i k y\left(\frac{\partial}{\partial x}+i \frac{\partial}{\partial y}\right) .
$$

(iii) There is a Fourier polynomial $P_{f}(\tau)=\sum_{-\infty \ll n \leq 0} c_{f}^{+}(n) q^{n} \in \mathbb{C}\left[q^{-1}\right]$ such that $f(\tau)=P_{f}(\tau)+O\left(e^{-\varepsilon y}\right)$ as $y \rightarrow \infty$ for some $\varepsilon>0$. Analogous conditions are required at all cusps.

Let $H_{k}\left(\Gamma_{0}(4 m)\right)$ be the space of such harmonic weak Maass forms. This space can be denoted by $H_{k}^{+}\left(\Gamma_{0}(4 m)\right)$ in the context of [5], which is the inverse image of $S_{2-k}\left(\Gamma_{0}(4 m)\right)$ under a certain differential operator $\xi_{k}$. Note that $M_{k}^{!}\left(\Gamma_{0}(4 m)\right) \subset$ $H_{k}\left(\Gamma_{0}(4 m)\right)$. The polynomial $P_{f} \in \mathbb{C}\left[q^{-1}\right]$ is called the principal part of $f$ at the corresponding cusps.

2.2. Vector valued modular forms. Let $\mathrm{Mp}_{2}(\mathbb{R})$ be the metaplectic two-fold cover of $\mathrm{SL}_{2}(\mathbb{R})$. Thus elements of $\mathrm{Mp}_{2}(\mathbb{R})$ are pairs $(M, \phi)$, where $M=\left(\begin{array}{ll}a & b \\ c & d\end{array}\right) \in$ $\mathrm{SL}_{2}(\mathbb{R})$ and $\phi: \mathbb{H} \rightarrow \mathbb{C}$ is a holomorphic function with $\phi(\tau)^{2}=c \tau+d$. The multiplication is defined by

$$
(M, \phi(\tau))\left(M^{\prime}, \phi^{\prime}(\tau)\right)=\left(M M^{\prime}, \phi\left(M^{\prime} \tau\right) \phi^{\prime}(\tau)\right) .
$$

For $M=\left(\begin{array}{ll}a & b \\ c & d\end{array}\right) \in \mathrm{SL}_{2}(\mathbb{R})$ put $\tilde{M}:=\left(\left(\begin{array}{ll}a & b \\ c & d\end{array}\right), \sqrt{c \tau+d}\right) \in \mathrm{Mp}_{2}(\mathbb{R})$ and let $\operatorname{Mp}_{2}(\mathbb{Z})$ be the integral metaplectic group, that is, the inverse image of $\mathrm{SL}_{2}(\mathbb{Z})$ under the 
covering map $\mathrm{Mp}_{2}(\mathbb{R}) \rightarrow \mathrm{SL}_{2}(\mathbb{R})$. It is well known that $\mathrm{Mp}_{2}(\mathbb{Z})$ is generated by $T:=\left(\left(\begin{array}{ll}1 & 1 \\ 0 & 1\end{array}\right), 1\right)$ and $S:=\left(\left(\begin{array}{cc}0 & -1 \\ 1 & 0\end{array}\right), \sqrt{\tau}\right)$.

Let $(V, Q)$ be a nondegenerate rational quadratic space of signature $\left(b^{+}, b^{-}\right)$. Let $L \subset V$ be an even lattice with dual $L^{\prime}$ and denote the standard basis elements of the group algebra $\mathbb{C}\left[L^{\prime} / L\right]$ by $\mathfrak{e}_{\gamma}$ for $\gamma \in L^{\prime} / L$, and write $\langle\cdot, \cdot\rangle$ for the standard scalar product, anti-linear in the second entry, such that $\left\langle\mathfrak{e}_{\gamma}, \mathfrak{e}_{\gamma^{\prime}}\right\rangle=\delta_{\gamma, \gamma^{\prime}}$. There is a unitary representation $\rho_{L}$ of $\mathrm{Mp}_{2}(\mathbb{Z})$ on $\mathbb{C}\left[L^{\prime} / L\right]$, the so-called Weil representation, which is defined by

$$
\begin{aligned}
\rho_{L}(T)\left(\mathfrak{e}_{\gamma}\right) & :=e(Q(\gamma)) \mathfrak{e}_{\gamma}, \\
\rho_{L}(S)\left(\mathfrak{e}_{\gamma}\right) & :=\frac{e\left(\left(b^{-}-b^{+}\right) / 8\right)}{\sqrt{\left|L^{\prime} / L\right|}} \sum_{\delta \in L^{\prime} / L} e(-(\gamma, \delta)) \mathfrak{e}_{\delta},
\end{aligned}
$$

where $e(z):=e^{2 \pi i z}$ and $(X, Y):=Q(X+Y)-Q(X)-Q(Y)$ is the associated bilinear form. We denote by $\bar{\rho}_{L}$ the dual representation of $\rho_{L}$.

Let $k \in \frac{1}{2} \mathbb{Z}$. A holomorphic function $f: \mathbb{H} \rightarrow \mathbb{C}\left[L^{\prime} / L\right]$ is called a weakly holomorphic modular form of weight $k$ and type $\rho_{L}$ for the group $\mathrm{Mp}_{2}(\mathbb{Z})$ if it satisfies:

(i) $f(M \tau)=\phi(\tau)^{2 k} \rho_{L}(M, \phi) f(\tau)$ for all $(M, \phi) \in \mathrm{Mp}_{2}(\mathbb{Z})$;

(ii) $f$ is meromorphic at the cusp $\infty$.

Here condition (ii) means that $f$ has a Fourier expansion of the form

$$
f(\tau)=\sum_{\gamma \in L^{\prime} / L} \sum_{\substack{n \in \mathbb{Z}+Q(\gamma) \\ n \gg-\infty}} c_{f}(\gamma, n) e(n \tau) \mathfrak{e}_{\gamma} .
$$

The space of these $\mathbb{C}\left[L^{\prime} / L\right]$-valued weakly holomorphic modular forms is denoted by $M_{k, \rho_{L}}^{!}$. Similarly, we can define the space $M_{k, \bar{\rho}_{L}}^{!}$of $\mathbb{C}\left[L^{\prime} / L\right]$-valued weakly holomorphic modular forms of type $\bar{\rho}_{L}$.

A smooth function $f: \mathbb{H} \rightarrow \mathbb{C}\left[L^{\prime} / L\right]$ is called a harmonic weak Maass form of weight $k$ and type $\rho_{L}$ for the group $\mathrm{Mp}_{2}(\mathbb{Z})$ if it satisfies the following conditions:

(i) $f(M \tau)=\phi(\tau)^{2 k} \rho_{L}(M, \phi) f(\tau)$ for all $(M, \phi) \in \mathrm{Mp}_{2}(\mathbb{Z})$.

(ii) $\Delta_{k} f=0$.

(iii) There is a Fourier polynomial $P_{f}(\tau)=\sum_{\gamma \in L^{\prime} / L} \sum_{\substack{n \in \mathbb{Z}+Q(\gamma) \\-\infty \ll n \leq 0}} c_{f}^{+}(\gamma, n) e(n \tau) \mathfrak{e}_{\gamma}$ such that $f(\tau)=P_{f}(\tau)+O\left(e^{-\varepsilon y}\right)$ as $y \rightarrow \infty$ for some $\varepsilon>0$.

Let $H_{k, \rho_{L}}$ be the space of these $\mathbb{C}\left[L^{\prime} / L\right]$-valued harmonic weak Maass forms. This space is denoted by $H_{k, L}^{+}$in [5], which is the inverse image of $S_{2-k, L^{-}}$under $\xi_{k}$. Note that $M_{k, \rho_{L}}^{!} \subset H_{k, \rho_{L}}$. We define the space $H_{k, \bar{\rho}_{L}}$ in an analogous manner. In particular, $f \in H_{k, \rho_{L}}$ has a unique decomposition of the form $f=f^{+}+f^{-}$with

$$
\begin{aligned}
f^{+}(\tau) & =\sum_{\gamma \in L^{\prime} / L} \sum_{\substack{n \in \mathbb{Z}+Q(\gamma) \\
n \gg-\infty}} c_{f}^{+}(\gamma, n) e(n \tau) \mathfrak{e}_{\gamma}, \\
f^{-}(\tau) & =\sum_{\gamma \in L^{\prime} / L} \sum_{\substack{n \in \mathbb{Z}+Q(\gamma) \\
n<0}} c_{f}^{-}(\gamma, n) \Gamma(1-k, 4 \pi|n| y) e(n \tau) \mathfrak{e}_{\gamma} .
\end{aligned}
$$

2.3. Harmonic Maass-Jacobi forms. In this section we mostly follow the notation in [2]. 
Definition 3. A function $\phi: \mathbb{H} \times \mathbb{C} \rightarrow \mathbb{C}$ is a harmonic Maass-Jacobi form of weight $k$ and index $m$ if $\phi$ is real analytic in $\tau=x+i y \in \mathbb{H}$ and $z=u+i v \in \mathbb{C}$ satisfying the following conditions:

(1) For all $A=\left[\left(\begin{array}{ll}a & b \\ c & d\end{array}\right),(\lambda, \mu)\right] \in \mathrm{SL}_{2}(\mathbb{Z}) \ltimes \mathbb{Z}^{2}$,

$$
\phi\left(\frac{a \tau+b}{c \tau+d}, \frac{z+\lambda \tau+\mu}{c \tau+d}\right)(c \tau+d)^{-k} e^{2 \pi i m\left(-\frac{c(z+\lambda \tau+\mu)^{2}}{c \tau+d}+\lambda^{2} \tau+2 \lambda z\right)}=\phi(\tau, z) .
$$

(2) $C^{k, m}(\phi)=0$, where $C^{k, m}$ is the Casimir element of the real Jacobi group (see p. 2305 in [2]).

(3) $\phi(\tau, z)=O\left(e^{a y} e^{2 \pi m v^{2} / y}\right)$ as $y \rightarrow \infty$ for some $a>0$.

Let $\widehat{\mathbb{J}}_{k, m}$ be the space of harmonic Maass-Jacobi forms of weight $k$ and index $m$ which are holomorphic in $z$. In fact, we are interested in the subspace $\widehat{\mathbb{J}}_{k, m}^{c u s p}$ consisting of the elements $\phi \in \widehat{\mathbb{J}}_{k, m}$ whose Fourier expansion is of the form

$$
\phi(\tau, z)=\sum_{\substack{n, r \in \mathbb{Z} \\ D \ll \infty}} c^{+}(n, r) q^{n} \zeta^{r}+\sum_{\substack{n, r \in \mathbb{Z} \\ D>0}} c^{-}(n, r) \Gamma\left(\frac{3}{2}-k, \frac{\pi D y}{m}\right) q^{n} \zeta^{r},
$$

where $q=e(\tau)$ and $\zeta=e(z)$. The space $\widehat{\mathbb{J}}_{k, m}^{c u s p}$ is in fact the inverse image of $J_{3-k, m}^{s k, c u s p}$ under a certain differential operator $\xi_{k, m}$ (see [2, Remarks (1) on p. 2307]).

\section{Proof of Theorem 1}

We give a proof only of (1) since (2) can be argued in a similar way. First, we prove that for $f \in H_{k+\frac{1}{2}}^{+}\left(\Gamma_{0}(4 m)\right)$ the $\mathbb{C}\left[L^{\prime} / L\right]$-valued function $F$ as defined in (1.3) belongs to $H_{k+\frac{1}{2}, \rho_{L}}$. By inspecting the Fourier expansion of $f$ with the forms in (1.1) and (1.2) we get that $f(\tau)=\sum_{\gamma \in L^{\prime} / L} F_{\gamma}(4 m \tau)$. Since it is straightforward to check that $F(\tau+1)=\rho_{L}(T) F(\tau)$, we only need to show that

$$
F\left(-\frac{1}{\tau}\right)=\tau^{k+\frac{1}{2}} \rho_{L}(S) F(\tau) .
$$

We claim that (3.1) holds for any prime $m$ or $m=1$ by extending the argument in [8, pp. 735-737], where it was proved for

$$
m \in \mathfrak{S}:=\{2,3,5,7,11,13,17,19,23,29,31,41,47,59,71\} .
$$

For $j$ prime to $4 m$ set

$$
f_{j}:=\left.\left.f\right|_{k+\frac{1}{2}}\left(\left(\begin{array}{cc}
1 & j \\
0 & 4 m
\end{array}\right),(4 m)^{1 / 4}\right)\right|_{k+\frac{1}{2}} W_{4 m},
$$

where the slash operator is defined as

$$
\left.f\right|_{k+\frac{1}{2}}(A, \psi(\tau))=\psi(\tau)^{-2 k-1} f(A \tau)
$$

(see [8]), and

$$
W_{4 m}:=\left(\left(\begin{array}{cc}
0 & -1 \\
4 m & 0
\end{array}\right),(4 m)^{1 / 4} \sqrt{-i \tau}\right) .
$$

Choose $b, d \in \mathbb{Z}$ so that $j d-4 m b=1$. Then one finds that

$$
\begin{aligned}
\left(\left(\begin{array}{cc}
1 & j \\
0 & 4 m
\end{array}\right),(4 m)^{1 / 4}\right) W_{4 m} & =\left(\left(\begin{array}{cc}
4 m j & -1 \\
16 m^{2} & 0
\end{array}\right), 2 \sqrt{-m i \tau}\right) \\
& =(M, J(M, \tau))\left(\left(\begin{array}{cc}
4 m & -d \\
0 & 4 m
\end{array}\right), \psi_{j}\right),
\end{aligned}
$$

where

$$
\psi_{j}:=\left(\frac{4 m}{j}\right) \sqrt{\left(\frac{-1}{j}\right)} e(-1 / 8),
$$


$M=\left(\begin{array}{cc}j & b \\ 4 m & d\end{array}\right)$ and $J(M, \tau)$ denotes the automorphy factor for the theta function $\sum_{n \in \mathbb{Z}} q^{n^{2}}$, that is,

$$
J(M, \tau):=\left(\frac{c}{d}\right) \varepsilon_{d}^{-1}(c \tau+d)^{\frac{1}{2}}, \quad M=\left(\begin{array}{cc}
* & * \\
c d
\end{array}\right) \in \Gamma_{0}(4 m) ;
$$

here $\left(\frac{c}{d}\right)$ is the usual Jacobi symbol.

This implies that

$$
\begin{aligned}
f_{j} & =\left.f\right|_{k+\frac{1}{2}}\left(\left(\begin{array}{cc}
1 & j \\
0 & 4 m
\end{array}\right),(4 m)^{1 / 4}\right) W_{4 m} \\
& =\psi_{j}^{-2 k-1} f\left(\tau-\frac{j^{-1}}{4 m}\right) \\
& =\psi_{j}^{-2 k-1} \sum_{\gamma(2 m)} e\left(-\frac{j^{-1} \gamma^{2}}{4 m}\right) F_{\gamma}(4 m \tau),
\end{aligned}
$$

where $j^{-1}$ denotes an integer which is the inverse of $j$ in $(\mathbb{Z} / 4 m \mathbb{Z})^{\times}$. On the other hand, we have by definition that

$$
\begin{aligned}
f_{j} & =\left.(4 m)^{(-2 k-1) / 4}\left(\sum_{\gamma(2 m)} e\left(\frac{j \gamma^{2}}{4 m}\right) F_{\gamma}\right)\right|_{k+\frac{1}{2}} W_{4 m} \\
& =(4 m)^{(-2 k-1) / 2} \sqrt{-i \tau}^{-2 k-1} \sum_{\gamma(2 m)} e\left(\frac{j \gamma^{2}}{4 m}\right) F_{\gamma}\left(-\frac{1}{4 m \tau}\right) .
\end{aligned}
$$

Replacing $\tau$ by $\tau / 4 m$ in (3.2) and (3.3), one has the following identity:

$$
\sum_{\gamma(2 m)} e\left(\frac{j \gamma^{2}}{4 m}\right) F_{\gamma}\left(-\frac{1}{\tau}\right)=\left(\frac{4 m}{j}\right) \sqrt{\left(\frac{-1}{j}\right)}^{-1} \tau^{k+\frac{1}{2}} \sum_{\gamma(2 m)} e\left(-\frac{j^{-1} \gamma^{2}}{4 m}\right) F_{\gamma}(\tau) \text {. }
$$

Let $R$ be a $2 m \times 2 m$ matrix defined by

$$
R:=\frac{e(-1 / 8)}{\sqrt{2 m}}\left(e\left(-\frac{l \gamma}{2 m}\right)\right)_{l(2 m), \gamma(2 m)} .
$$

In order to show $F \in H_{k+\frac{1}{2}, \rho_{L}}$ we first need to prove (3.1), i.e.

$$
\left(\begin{array}{c}
\vdots \\
F_{\gamma} \\
\vdots
\end{array}\right)(-1 / \tau)=\tau^{k+\frac{1}{2}} R\left(\begin{array}{c}
\vdots \\
F_{\gamma} \\
\vdots
\end{array}\right)(\tau) \text {. }
$$

Since $F_{\gamma}=F_{-\gamma}$, the above identity is equivalent to

$$
B\left(\begin{array}{c}
\vdots \\
F_{\gamma} \\
\vdots
\end{array}\right)(-1 / \tau)=\tau^{k+\frac{1}{2}} B R\left(\begin{array}{c}
\vdots \\
F_{\gamma} \\
\vdots
\end{array}\right)(\tau)
$$

for some matrix $B$ with $2 m$ columns of which the $m+1$ columns corresponding to $\gamma=0,1, \cdots, m$ are linearly independent. For instance, in 8$] B$ was chosen as

$$
A:=\left(e\left(\frac{\mathrm{J}_{\ell} \gamma^{2}}{4 m}\right)\right)_{\ell(\varphi(4 m)), \gamma(2 m)}
$$

and was verified that its rank is $m+1$ if $m \in \mathfrak{S}-\{2\}$. Here $\mathrm{J}_{\ell}$ is the $\ell$ th largest element in $\{j \mid 1 \leq j \leq 4 m,(j, 4 m)=1\}$. 
Here, we take $B$ as $C A$ with

$$
C:=\left(e\left(-\frac{\mathrm{J}_{\ell} \beta^{2}}{4 m}\right)\right)_{\beta(2 m), \ell(\varphi(4 m))}
$$

and state the following lemma:

Lemma 4. For $m=1$ or an odd prime, the rank of $B=\left(b_{\beta \gamma}\right)_{\beta(2 m), \gamma(2 m)}:=C A$ is $m+1$, and the $m+1$ columns of $B$ corresponding to $\gamma=0,1, \cdots, m$ are linearly independent.

Proof. Note that the $\beta \gamma$-th entry $b_{\beta \gamma}$ with $0 \leq \beta, \gamma<2 m$ is given by

$$
\begin{aligned}
b_{\beta \gamma} & =\sum_{\mathrm{J}(4 m)^{*}} e\left(\frac{\mathrm{J}\left(\gamma^{2}-\beta^{2}\right)}{4 m}\right) \\
& = \begin{cases}2 \varphi(m) & \text { if } \beta \equiv \pm \gamma \bmod 2 m, \beta \equiv \gamma \bmod 2, \\
-2 & \text { if } \beta \not \equiv \pm \gamma \bmod 2 m, \beta \\
0 & \text { if } \beta \not \equiv \gamma \bmod 2 .\end{cases}
\end{aligned}
$$

For example, if $\beta \not \equiv \gamma \bmod 2$, then $e\left(\frac{\mathrm{J}\left(\gamma^{2}-\beta^{2}\right)}{4 m}\right)+e\left(\frac{(\mathrm{J}+2 m)\left(\gamma^{2}-\beta^{2}\right)}{4 m}\right)=0$ for each $\mathrm{J} \in(\mathbb{Z} / 4 m \mathbb{Z})^{\times}$, and hence $b_{\beta \gamma}=0$. So, $B$ is symmetric and $b_{\beta, \gamma}=b_{\beta, 2 m-\gamma}$ for all $0 \leq \beta, \gamma<2 m$ with $\gamma \neq 0$. More precisely, $B$ is of the form

$$
\left(\begin{array}{cccccccc}
2 \varphi(m) & 0 & -2 & 0 & \cdots & 0 & -2 & 0 \\
0 & 2 \varphi(m) & 0 & -2 & \cdots & -2 & 0 & 2 \varphi(m) \\
-2 & 0 & 2 \varphi(m) & 0 & \cdots & 0 & 2 \varphi(m) & 0 \\
0 & -2 & 0 & 2 \varphi(m) & \cdots & 2 \varphi(m) & 0 & -2 \\
\vdots & \vdots & \vdots & \vdots & \ddots & \vdots & \vdots & \vdots \\
0 & -2 & 0 & 2 \varphi(m) & \cdots & 2 \varphi(m) & 0 & -2 \\
-2 & 0 & 2 \varphi(m) & 0 & \cdots & 0 & 2 \varphi(m) & 0 \\
0 & 2 \varphi(m) & 0 & -2 & \cdots & -2 & 0 & 2 \varphi(m)
\end{array}\right) .
$$

By subtracting either the first or the second column from the other columns, it is easy to infer that the upper-left $(m+1) \times(m+1)$ minor of $B$; i.e. the submatrix of $B$ with $0 \leq \beta, \gamma \leq m$ is nonsingular.

Using the Gauss sum formula (see [8, p. 736]), we note that

$$
A R=\left(\left(\frac{4 m}{\mathrm{~J}_{\ell}}\right){\sqrt{\left(\frac{-1}{\mathrm{~J}_{\ell}}\right)}}^{-1} e\left(-\frac{\mathrm{J}_{\ell}^{-1} \gamma^{2}}{4 m}\right)\right)_{\ell(\varphi(4 m)), \gamma(2 m)} .
$$

So the equality in (3.4) is equivalent to

$$
A\left(\begin{array}{c}
\vdots \\
F_{\gamma} \\
\vdots
\end{array}\right)(-1 / \tau)=\tau^{k+\frac{1}{2}} A R\left(\begin{array}{c}
\vdots \\
F_{\gamma} \\
\vdots
\end{array}\right)(\tau)
$$

which implies that

$$
B\left(\begin{array}{c}
\vdots \\
F_{\gamma} \\
\vdots
\end{array}\right)(-1 / \tau)=\tau^{k+\frac{1}{2}} B R\left(\begin{array}{c}
\vdots \\
F_{\gamma} \\
\vdots
\end{array}\right)(\tau)
$$


Combining this with Lemma 4, we see that $F$ satisfies the transformation property (3.1) for any prime $m>2$ and $m=1$. From (3.2), (3.4), and Lemma 4 we can note that each $\left.F_{\gamma}\right|_{k+\frac{1}{2}}\left(\left(\begin{array}{cc}0 & -1 \\ 1 & 0\end{array}\right), \sqrt{\tau}\right)$ can be written as a linear combination of

$$
f_{j}:=\left.\left.f\right|_{k+\frac{1}{2}}\left(\left(\begin{array}{cc}
1 & j \\
0 & 4 m
\end{array}\right),(4 m)^{1 / 4}\right)\right|_{k+\frac{1}{2}} W_{4 m}
$$

for all $(j, 4 m)=1$. Since $\Delta_{k+\frac{1}{2}}$ commutes with the Petersson slash operator (see [9]), each $\Delta_{k+\frac{1}{2}} F_{\gamma}$ vanishes, i.e. $F \in H_{k+\frac{1}{2}, \rho_{L}}$ for $m \neq 2$.

The same procedure as given in [8, Remark 3.2] can be applied to the case when $m=2$, so we omit the detailed proof.

In order to verify the isomorphism, we next consider the converse. For a given $F=\sum_{\gamma(2 m)} F_{\gamma} \mathfrak{e}_{\gamma} \in H_{k+\frac{1}{2}, \rho_{L}}$ define

$$
f(\tau):=\sum_{\gamma(2 m)} F_{\gamma}(4 m \tau)
$$

A straightforward computation shows that $f$ satisfies the condition $c_{f}^{ \pm}(n)=0$ unless $(-1)^{k} n \equiv \square \bmod 4 m$ by inspecting the Fourier expansion of $F$. Also $\Delta_{k+\frac{1}{2}} f=0$ by (3.5). Thus it remains to show that

$$
f\left(\frac{a \tau+b}{c \tau+d}\right)=\left(\frac{c}{d}\right) \sqrt{\left(\frac{-1}{d}\right)}^{-1}(c \tau+d)^{k+\frac{1}{2}} f(\tau)
$$

for all $\left(\begin{array}{ll}a & b \\ c & d\end{array}\right) \in \Gamma_{0}(4 m)$. We may assume that $d>0$ by multiplying $\left(\begin{array}{cc}-1 & 0 \\ 0 & -1\end{array}\right)$ if necessary.

Remark 3. In what follows, our results hold for arbitrary $m>0$. In fact one may apply our argument even for somewhat general discriminant forms $\left(L^{\prime} / L, Q\right)$.

Lemma 5. For any $n \in \mathbb{Z}$ one has

$$
\rho_{L} \widetilde{\left(\begin{array}{ll}
1 & 0 \\
n & 1
\end{array}\right)} \sum_{\gamma \in L^{\prime} / L} \mathfrak{e}_{\gamma}=\sum_{\gamma \in L^{\prime} / L} \mathfrak{e}_{\gamma} .
$$

Proof. Since $\widetilde{\left(\begin{array}{ll}1 & 0 \\ n & 1\end{array}\right)}=\widetilde{\left(\begin{array}{ll}1 & 0 \\ 1 & 1\end{array}\right)^{n}}$, it suffices to prove that

$$
\rho_{L} \widetilde{\left(\begin{array}{ll}
1 & 0 \\
1 & 1
\end{array}\right)} \sum_{\gamma \in L^{\prime} / L} \mathfrak{e}_{\gamma}=\sum_{\gamma \in L^{\prime} / L} \mathfrak{e}_{\gamma} .
$$

For $M \in \mathrm{SL}_{2}(\mathbb{Z})$ we define the coefficients $\rho_{\beta \gamma}(\tilde{M})$ of the representation $\rho_{L}$ by $\rho_{\beta \gamma}(\tilde{M})=\left\langle\rho_{L}(\tilde{M}) \mathfrak{e}_{\gamma}, \mathfrak{e}_{\beta}\right\rangle$. From Shintani's result [3, Proposition 1.1] for $\rho_{\beta \gamma}(\tilde{M})$ one has

$$
\begin{aligned}
\sum_{\gamma \in L^{\prime} / L} \rho_{\beta \gamma} \widehat{\left(\begin{array}{ll}
1 & 0 \\
1 & 1
\end{array}\right)} & =\sum_{\gamma \in L^{\prime} / L} \frac{e\left(\left(b^{-}-b^{+}\right) / 8\right)}{\sqrt{2 m}} e(Q(\beta)-(\beta, \gamma)+Q(\gamma)) \\
& =\frac{e\left(\left(b^{-}-b^{+}\right) / 8\right)}{\sqrt{2 m}} \sum_{\gamma \in L^{\prime} / L} e(Q(\beta-\gamma)) \\
& =1 .
\end{aligned}
$$

The last equality is from Milgram's formula (see [1]), which states that

$$
\sum_{\gamma \in L^{\prime} / L} e(Q(\gamma))=\sqrt{\left|L^{\prime} / L\right|} e\left(\left(b^{+}-b^{-}\right) / 8\right) .
$$


First notice that

$$
f(\tau)=\left\langle F(4 m \tau), \sum_{\gamma \in L^{\prime} / L} \mathfrak{e}_{\gamma}\right\rangle .
$$

Since $\left(\begin{array}{cc}4 m & 0 \\ 0 & 1\end{array}\right)\left(\begin{array}{ll}a & b \\ c & d\end{array}\right)=\left(\begin{array}{cc}a & 4 m b \\ c / 4 m & d\end{array}\right)\left(\begin{array}{cc}4 m & 0 \\ 0 & 1\end{array}\right)$, we get for $\left(\begin{array}{ll}a & b \\ c & d\end{array}\right) \in \Gamma_{0}(4 m)$ that

$$
\begin{aligned}
& f\left(\frac{a \tau+b}{c \tau+d}\right)=\left\langle F\left(\left(\begin{array}{cc}
a & 4 m b \\
c / 4 m & d
\end{array}\right)(4 m \tau)\right), \sum_{\gamma \in L^{\prime} / L} \mathfrak{e}_{\gamma}\right\rangle
\end{aligned}
$$

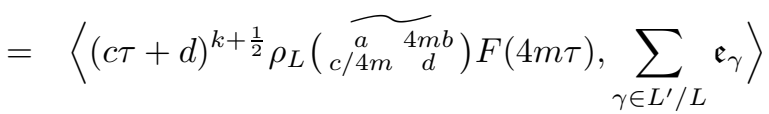

$$
\begin{aligned}
& =(c \tau+d)^{k+\frac{1}{2}}\left\langle F(4 m \tau), \rho_{L}\left(\underset{c / 4 m d}{\stackrel{a}{a m b})^{-1}} \sum_{\gamma \in L^{\prime} / L} \mathfrak{e}_{\gamma}\right\rangle .\right.
\end{aligned}
$$

Observe that $\Gamma^{0}(4 m)=\left\langle\Gamma_{0}(4 m) \cap \Gamma^{0}(4 m),\left(\begin{array}{ll}1 & 0 \\ 1 & 1\end{array}\right)\right\rangle$. More precisely, one has

$$
\left(\begin{array}{cc}
a & 4 m b \\
c / 4 m & d
\end{array}\right)=\left(\begin{array}{cc}
a(1-b c) & 4 m b \\
-4 m b(c / 4 m)^{2} & d
\end{array}\right)\left(\begin{array}{cc}
1 & 0 \\
a c / 4 m & 1
\end{array}\right) .
$$

We first consider the case $a>0$. The consistency condition implies that

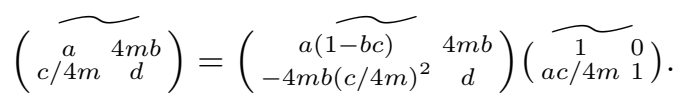

Since $\rho_{L}\left(\widetilde{\left.\begin{array}{c}1 \\ a c / 4 m\end{array}\right)}\right)_{\gamma \in L^{\prime} / L} \mathfrak{e}_{\gamma}=\sum_{\gamma \in L^{\prime} / L} \mathfrak{e}_{\gamma}$ by Lemma 5 , one finds from Borcherds' result [1, Theorem 5.4] that

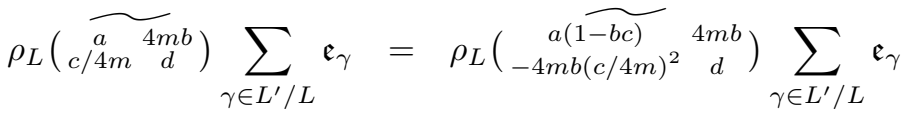

$$
\begin{aligned}
& =\left(\left(\frac{-4 m b}{d}\right) \sqrt{\left(\frac{-1}{d}\right)}^{-1}\right)^{b^{+}-b^{-}+\left(\frac{-1}{2 m}\right)-1}\left(\frac{d}{4 m}\right) \sum_{\gamma \in L^{\prime} / L} \mathfrak{e}_{\gamma} \\
& =\left(\frac{c}{d}\right) \sqrt{\left(\frac{-1}{d}\right)}^{-\left(\frac{-1}{m}\right)}\left(\frac{m}{d}\right)\left(\frac{d}{m}\right) \sum_{\gamma \in L^{\prime} / L} \mathfrak{e}_{\gamma} .
\end{aligned}
$$

Because $\sqrt{\left(\frac{-1}{d}\right)}^{1-\left(\frac{-1}{m}\right)}\left(\frac{m}{d}\right)\left(\frac{d}{m}\right)=1$, we get the following identity:

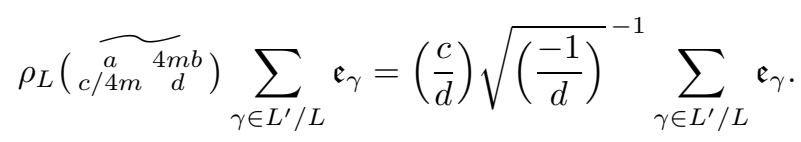

We claim that (3.7) is true for the case $a<0$. If $c=0$, then $a=d=-1$, and thereby it is straightforward to check (3.7). So we assume that $c \neq 0$. If we choose $x \in \mathbb{Z}$ such that $a+x c>0$, then with the elementary identity

$$
\left(\begin{array}{cc}
1 & 4 m x \\
0 & 1
\end{array}\right)\left(\begin{array}{cc}
a & 4 m b \\
c / 4 m & d
\end{array}\right)=\left(\begin{array}{cc}
a+x c & 4 m(b+x d) \\
c / 4 m & d
\end{array}\right)
$$


one can see that (3.7) is true even for $a<0$. Now if we insert (3.7) into (3.6), we obtain

$$
\begin{aligned}
f\left(\frac{a \tau+b}{c \tau+d}\right) & =\left(\frac{c}{d}\right) \sqrt{\left(\frac{-1}{d}\right)}^{-1}(c \tau+d)^{k+\frac{1}{2}}\left\langle F(4 m \tau), \sum_{\gamma \in L^{\prime} / L} \mathfrak{e}_{\gamma}\right\rangle \\
& =\left(\frac{c}{d}\right) \sqrt{\left(\frac{-1}{d}\right)}^{-1}(c \tau+d)^{k+\frac{1}{2}} f(\tau) .
\end{aligned}
$$

This completes the proof of Theorem 1 .

\section{ACKNOWLEDGMENTS}

The authors would like to express hearty thanks to the anonymous referee for a careful reading of the manuscript and useful corrections, especially for pointing out an error in an earlier version of Lemma 4.

\section{REFERENCES}

[1] Richard E. Borcherds, Reflection groups of Lorentzian lattices, Duke Math. J. 104 (2000), no. 2, 319-366, DOI 10.1215/S0012-7094-00-10424-3. MR.1773561 (2001h:11086)

[2] Kathrin Bringmann and Olav K. Richter, Zagier-type dualities and lifting maps for harmonic Maass-Jacobi forms, Adv. Math. 225 (2010), no. 4, 2298-2315, DOI 10.1016/j.aim.2010.03.033. MR2680205(2011h:11052)

[3] Jan H. Bruinier, Borcherds products on $\mathrm{O}(2, l)$ and Chern classes of Heegner divisors, Lecture Notes in Mathematics, vol. 1780, Springer-Verlag, Berlin, 2002. MR 1903920 (2003h:11052)

[4] Jan Hendrik Bruinier and Michael Bundschuh, On Borcherds products associated with lattices of prime discriminant, Ramanujan J. 7 (2003), no. 1-3, 49-61, DOI 10.1023/A:1026222507219. Rankin memorial issues. MR2035791 (2005a:11057)

[5] Jan Hendrik Bruinier and Jens Funke, On two geometric theta lifts, Duke Math. J. 125 (2004), no. 1, 45-90, DOI 10.1215/S0012-7094-04-12513-8. MR2097357 (2005m:11089)

[6] Bumkyu Cho and Youngju Choie, Zagier duality for harmonic weak Maass forms of integral weight, Proc. Amer. Math. Soc. 139 (2011), no. 3, 787-797, DOI 10.1090/S0002-9939-201010751-7. MR2745632 (2012b:11066)

[7] Martin Eichler and Don Zagier, The theory of Jacobi forms, Progress in Mathematics, vol. 55, Birkhäuser Boston Inc., Boston, MA, 1985. MR.781735 (86j:11043)

[8] Chang Heon Kim, Traces of singular values and Borcherds products, Bull. London Math. Soc. 38 (2006), no. 5, 730-740, DOI 10.1112/S0024609306018789. MR2268356 (2007h:11052)

[9] H. Maass, Lectures on modular functions of one complex variable, 2nd ed., Tata Institute of Fundamental Research Lectures on Mathematics and Physics, vol. 29, Tata Institute of Fundamental Research, Bombay, 1983. With notes by Sunder Lal. MR.734485 (85g:11034)

[10] Ameya Pitale, Jacobi Maaß forms, Abh. Math. Semin. Univ. Hambg. 79 (2009), no. 1, 87-111, DOI 10.1007/s12188-008-0013-9. MR2541345 (2010f:11075)

[11] Don Zagier, Traces of singular moduli, Motives, polylogarithms and Hodge theory, Part I (Irvine, CA, 1998), Int. Press Lect. Ser., vol. 3, Int. Press, Somerville, MA, 2002, pp. 211244. MR.1977587(2004h:11037)

[12] S. Zwegers, Mock theta functions, Ph.D. thesis, Universiteit Utrecht, The Netherlands, 2002.

Department of Mathematics, Dongguk University-Seoul, 30 Pildong-Ro 1-gil, JungGu, Seoul, 100-715, Republic of Korea

E-mail address: bam@dongguk.edu

Department of Mathematics, Pohang Mathematics Institute (PMi), POSteCH, Pohang, Republic of Korea

E-mail address: yjc@postech.ac.kr 